تقدير التبخر-النتح المرجعي عن طريق التنبؤ بدرجات الحرارة باستخدام نموذج عشوائي



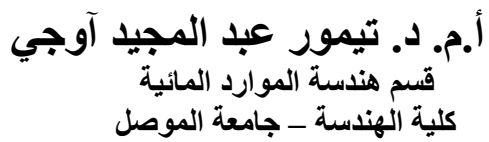

الخلاصـــة

تم في هذا البحث تطبيق النموذج العشوائي (ARIMA) لنمذجة قيم درجات الحرارة الثهرية لمنطقة الموصل

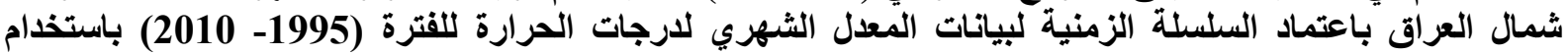



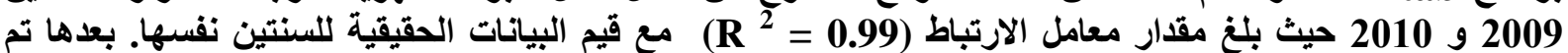

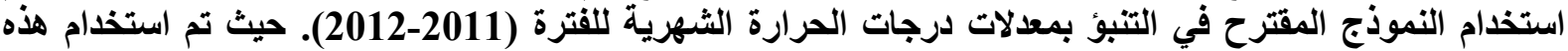



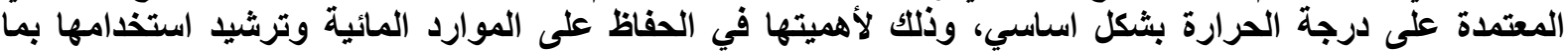


تقارباً كبيراً عند مقارنتها مع قيم التبخر-نتح باستخدام معادلة (Penman-Monteith)

\title{
Estimation of Reference Evapotranspiration by Predicting Temperature Values Using a Stochastic Model
}

\author{
Dr.Taymoor A. Awchi \\ Dept. of Water Resources Eng. \\ College of Eng./University of Mosul
}

\author{
Mr.Ihsan F. Hasan \\ Dams and Water Resources Research \\ Center/University of Mosul
}

\begin{abstract}
In this research, the stochastic model (ARIMA) was applied to modeling the monthly temperature values for the area of Mosul, Northern Iraq, by utilizing the timeseries data of monthly mean temperature for the period (1995-2010) using the Minitab Software. The performance of the proposed model has been approved through the prediction of monthly temperature values for the years 2009 and 2010 where the correlation coefficient value was $\left(R^{2}=0.99\right)$ with the values of the actual data for the same years. Then the proposed model was used to predict the mean monthly temperature values for the years (2011-2012). Due to its importance in the preservation of water resources and rational use in line with the future state of water in the region, this data was used to estimate the future values of Reference Evapotranspiration $\left(\mathbf{E T}_{\mathbf{0}}\right)$ using different empirical methods based essentially on temperature. The results of Blaney-Criddle and Hamon methods showed high correlation with $\mathbf{E T}_{\mathbf{0}}$ value calculated by Penman-Monteith model.
\end{abstract}

Key Words: Reference evapotranspiration, Temperature, ARIMA, Time series. 
يعتمد كل من التخطيط الاقتصادي والاداري لأنظمة الموارد المائية على دراسة التوقعات الهيدرولوجية لئية

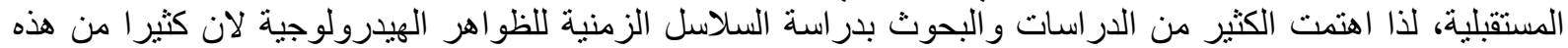

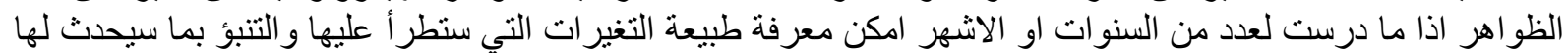

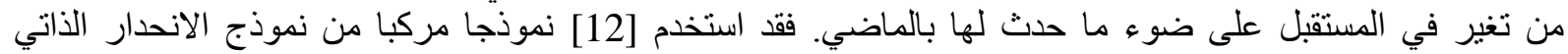

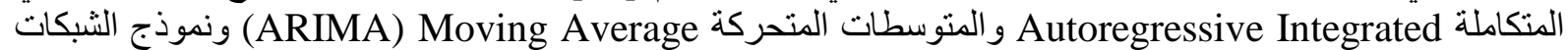

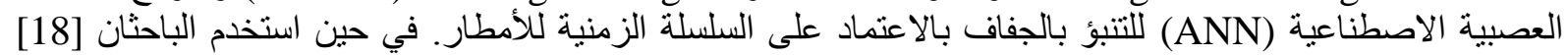

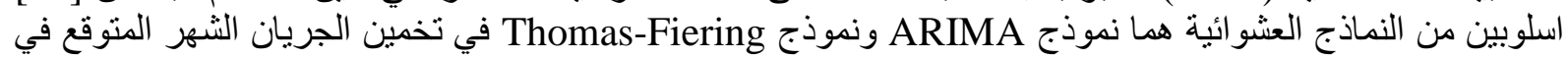

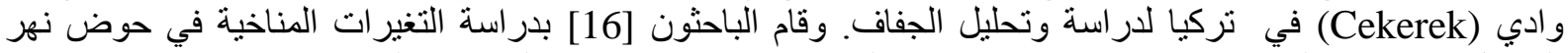

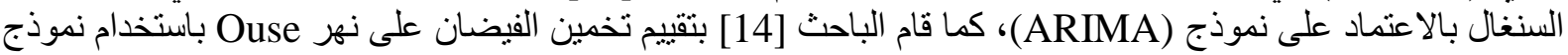





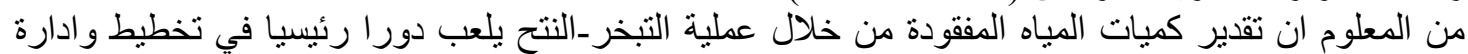

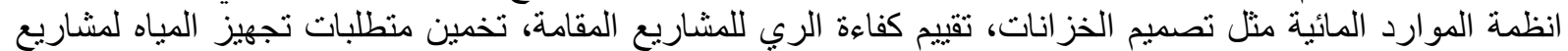

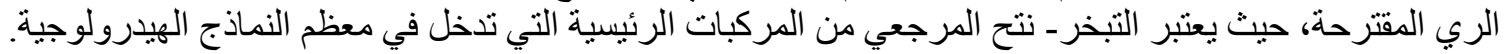

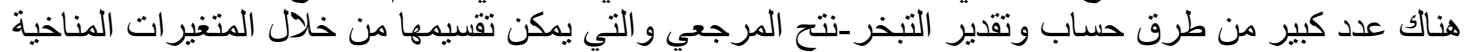

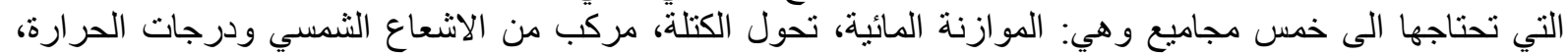

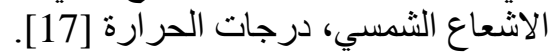
فقد قام العديد من الباحثين منذ منتصف القران القرن الماضي مثل [3,6,9,10,11] بجهود مضنية للتغلب على صعوبة الإنة

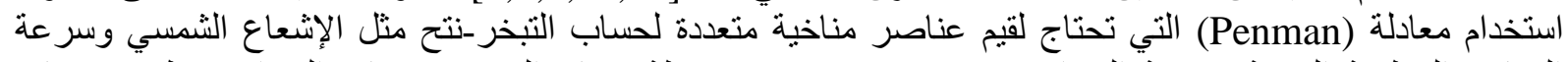

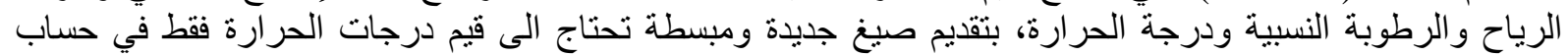
التبخر نتح المرجعي.

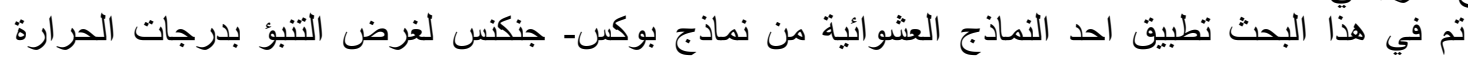

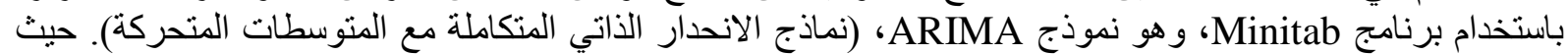

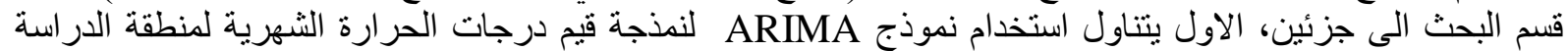

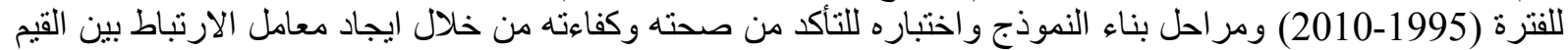

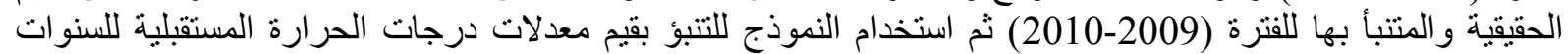


درجات الحرارة بشكل اساسي ومن ثم اختباره للتأكد من كفاءته.

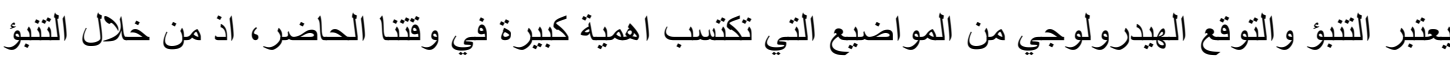

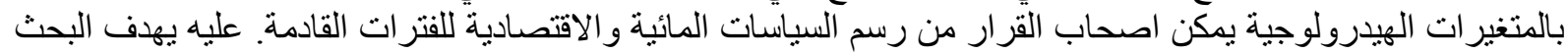

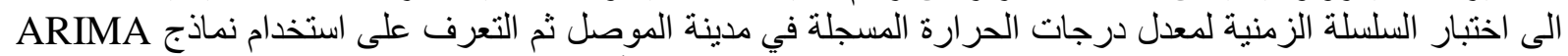

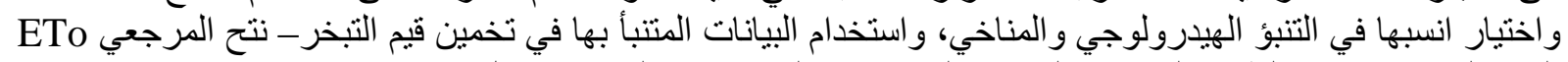

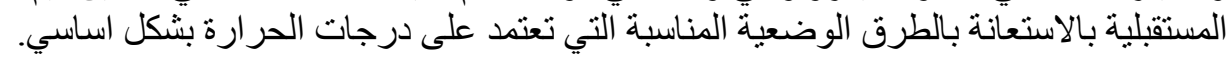

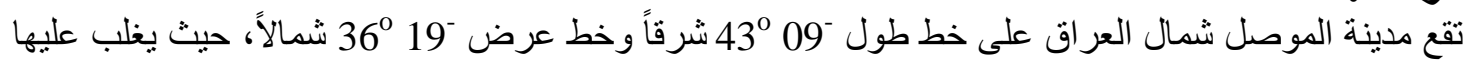



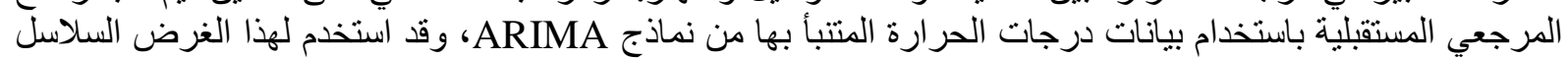

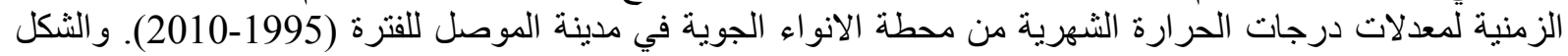
(1) يبين الموقع الجغر افي لمنطقة الدراسة. 


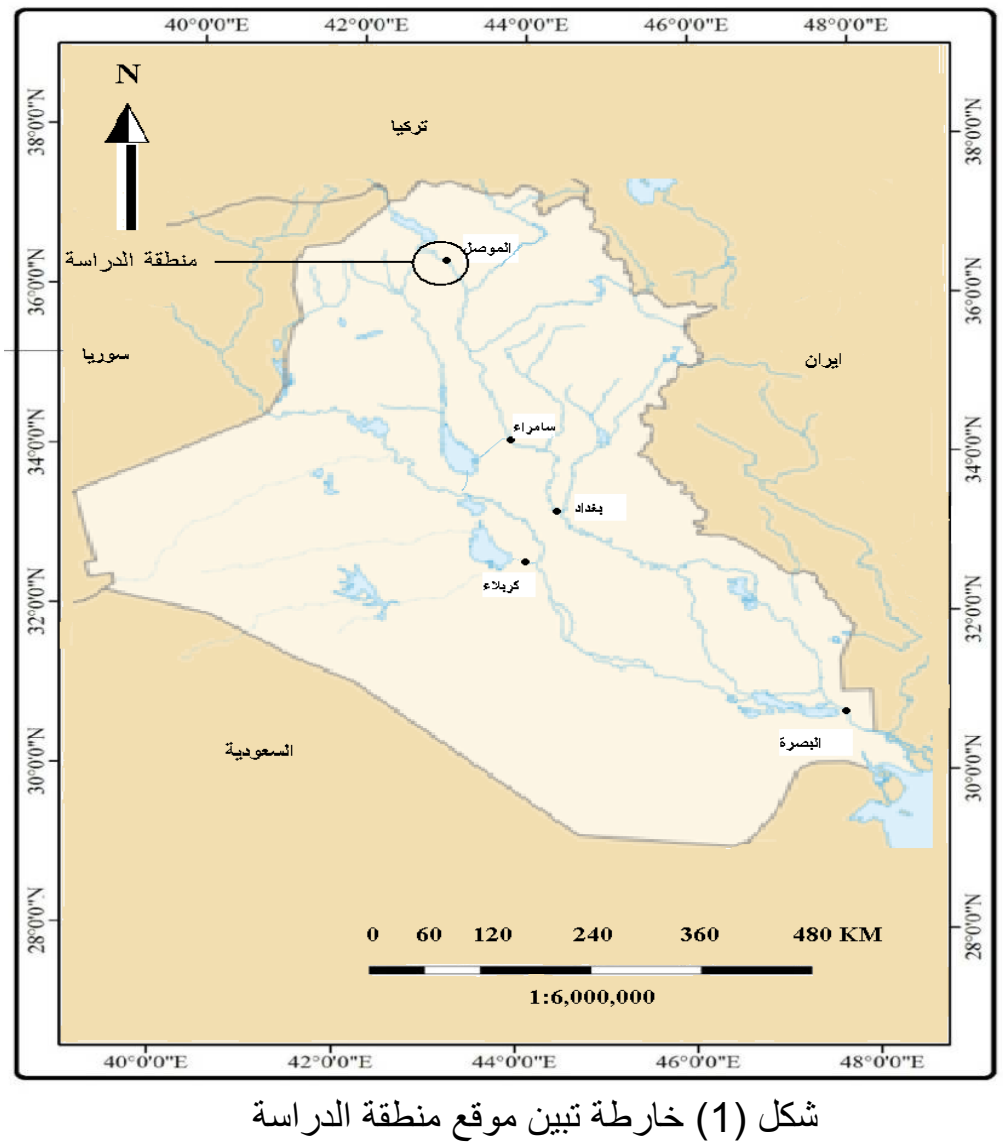

تستخدم النماذج العشو ائية Stochastic Models بشكل و اسع في تحليل ونمذجة السلاسل الزمنية الهيدرولوجية. تم في هذه الدراسة استخدام نماذج (ARIMA) في التنبؤ، ويقصد بنماذج (ARIMA) تلأك المنهجية التي طبقها كل من

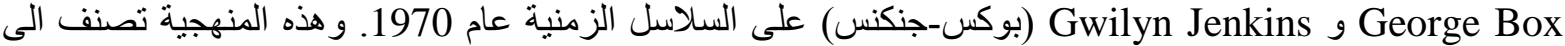

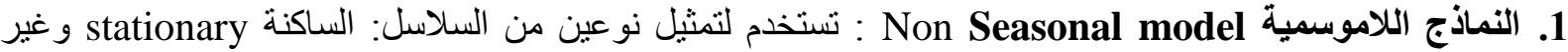
الساكنة Non-stationary ، وتعتبر السلسلة الزمنية ساكنة اذا كان لها وسط حسابي وتباين ثابتين. ومن هذه النماذج:

$\mathrm{X}_{\mathrm{t}}=\mu+\theta_{1} \mathrm{X}_{\mathrm{t}-1}+\theta_{2} \mathrm{X}_{\mathrm{t}-2}+\ldots+\theta_{\mathrm{p}} \mathrm{X}_{\mathrm{t}-\mathrm{p}}+\mathrm{e}_{\mathrm{t}}$ أــ نموذج الانحدار الأتي: Autoregressive model ويكتب بالثكل الآتي:

المتنبأ بها للفترة الزمنية (t) الحالية.


. 年

ويرمز لهذا النموذج بـ ( $A R(p$ حيث p pمثنل درجة النموذج. ويثير نموذج الانحدار الذاتي الى ان القيم

الحالية للمتغير X $X_{t-1}, X_{t-2}, X_{t-p}$

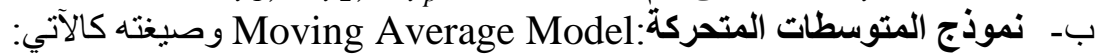
$X_{t}=\mu+e_{t}-\varphi_{1} e_{t-1}-\varphi_{2} e_{t-2}-\ldots . .-\varphi_{q} e_{t-q}$

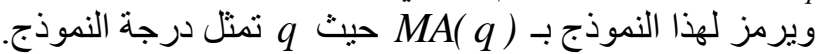


ج- نموذج الانحدار الذاتي و المتوسطات المتحركة ARMA(p,q حيث p,q تمثلان درجته. ويكتب بالصيغة الآتية [1] : $\mathrm{X}_{\mathrm{t}}=\mu+\theta_{1} \mathrm{X}_{\mathrm{t}-1}+\theta_{2} \mathrm{X}_{\mathrm{t}-2}+\ldots+\theta_{\mathrm{p}} \mathrm{X}_{\mathrm{t}-\mathrm{p}}+\mathrm{e}_{\mathrm{t}}-\varphi_{1} \mathrm{e}_{\mathrm{t}-1}-\varphi_{2} \mathrm{e}_{\mathrm{t}-2}-\ldots-\varphi_{\mathrm{q}} \mathrm{e}_{\mathrm{t}-\mathrm{q}}$


الفروق المناسبة للسلسلة الاصلية فمثلاً الفرق الأول يكون وفقاً للمعادلة الآتية:

$\mathrm{W}_{\mathrm{t}}=\mathrm{X}_{\mathrm{t}}-\mathrm{X}_{\mathrm{t}-1}$

وتختبر السلسلة الزمنيـة الجديدة فـاذا أصبحت سـاكنة تكون درجـة التفاضل d مسـاوية لـ1، و وإذا كانت السلسلة



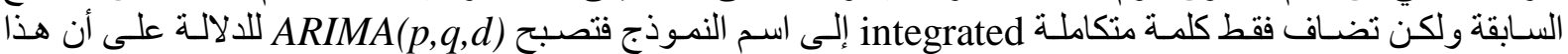
النموذج استخدم لتمثنيل سلسلة زمنية غير ساكنة.

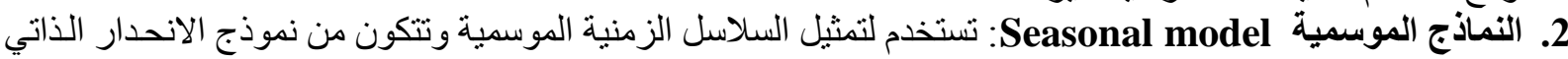
و المتوسطات المتحركة الموسمية

$\mathrm{X}_{\mathrm{t}}=\mu+\theta_{\mathrm{S}} \mathrm{X}_{\mathrm{t}-\mathrm{S}}+\theta_{2 \mathrm{~S}} \mathrm{X}_{\mathrm{t}-2 \mathrm{~S}}+\ldots+\theta_{\mathrm{PS}} \mathrm{X}_{\mathrm{t}-\mathrm{PS}}+\mathrm{e}_{\mathrm{t}}-\varphi_{\mathrm{S}} \mathrm{e}_{\mathrm{t}-\mathrm{S}}-\varphi_{2 \mathrm{~S}} \mathrm{e}_{\mathrm{t}-2 \mathrm{~S}}-\ldots-\varphi_{\mathrm{QS}} \mathrm{e}_{\mathrm{t}-\mathrm{QS}}$

أما إذا كانت السلاسل الموسمية غير ساكنة فتحول إلى ساكنة عن طريق أخذ الفرق الموسمي وفق المعادلة الآتية: $\mathrm{W}_{\mathrm{t}}=\mathrm{X}_{\mathrm{t}}-\mathrm{X}_{\mathrm{t}-\mathrm{S}}$

3. النموذج الموسـي المضـاعف ARIMA(p,q,d)×(P,Q,D) : هو خليط من النمـاذج اللاموسمية والموسمية ويكتب $\theta_{\mathrm{p}}(\mathrm{B}) \theta_{\mathrm{P}}\left(\mathrm{B}^{\mathrm{S}}\right) \nabla^{\mathrm{d}} \nabla_{\mathrm{s}}^{D} \mathrm{X}_{\mathrm{t}}=\varphi_{\mathrm{q}}(\mathrm{B}) \varphi_{\mathrm{Q}}\left(\mathrm{B}^{\mathrm{S}}\right) \mathrm{e}_{\mathrm{t}}$ Function (ACF) الموسمية و الموسمية الساكنة المختلفة.

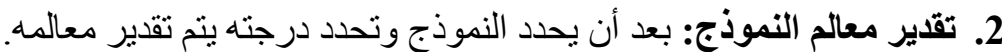
3. اختبارات دقة النتائج التنبؤية: هنالك اختبار ات عديدة تعتمد في تقييم النماذج المنتبأ بها ومنها الآتي [8]:

$\mathrm{MAE}=\frac{\sum\left|\mathrm{Y}_{\mathrm{t}}-\mathrm{X}_{\mathrm{t}}\right|}{\mathrm{n}}$

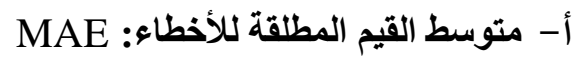
وتحسب بالثكل الآتي:

$\operatorname{MSE}=\frac{\sum\left(\mathrm{Y}_{\mathrm{t}}-\mathrm{X}_{\mathrm{t}}\right)^{2}}{\mathrm{n}}$

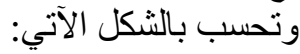

MAPE $=\frac{\sum\left(\frac{\left|Y_{t}-X_{t}\right|}{Y_{t}}\right)}{n}$ 
$\mathrm{MPE}=\frac{\sum\left(\frac{\left(\mathrm{Y}_{\mathrm{t}}-\mathrm{X}_{\mathrm{t}}\right)^{2}}{\mathrm{Y}_{\mathrm{t}}}\right)}{\mathrm{n}}$

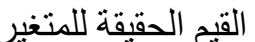

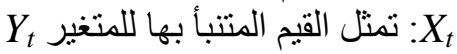
وفي جميع الحالات كلما كانت قيم اختبار ات الدقة التنبؤية اقل كانت الدقة التنبؤية للنموذج اكبر.

لغرض تطبيق نموذج ARIMA للتنبؤ بدرجات الحرارة تم أخذ البيانات الخاصة بدرجات الحرارة الثهرية للفترة

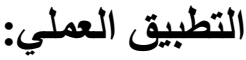

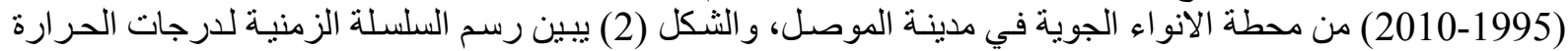

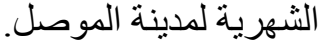

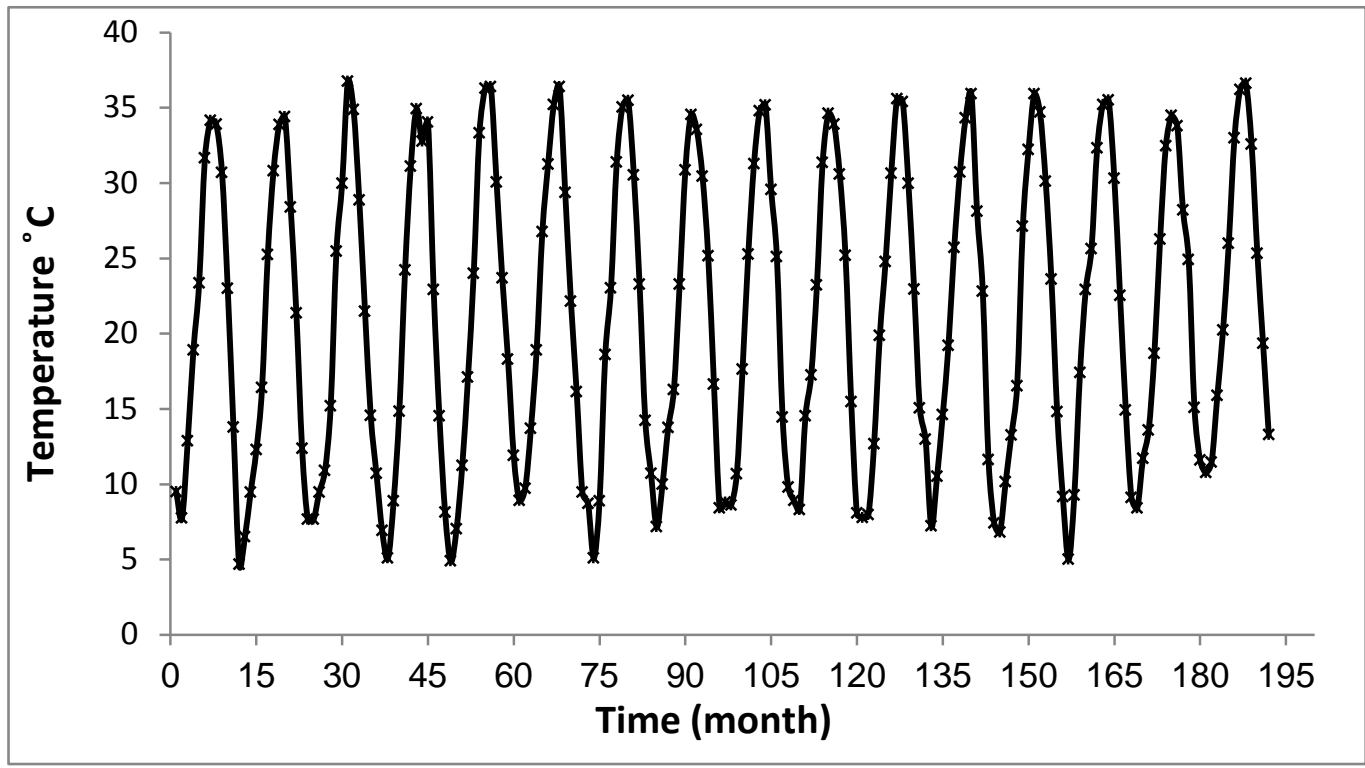

شكل (2) المعدلات الثهرية لدرجات الحرارة لمنطقة الدراسة خلال الفترة 1995-2010

ولغرض معرفة طييعة السلسلة الزمنية من حيث السكون تم إيجاد قيم معاملات الارتباط الذاتي (ACF) كما في الشكل

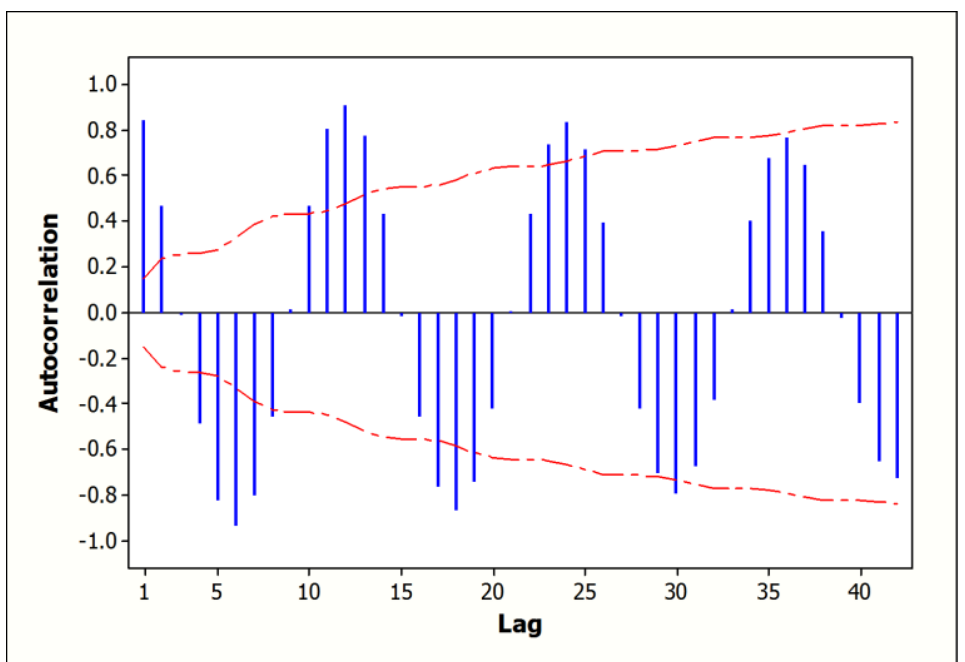

شكل (3) قيم معاملات الارتباط الذاتي ACF لسلسلة درجات الحر ارة في منطقة الدراسة 


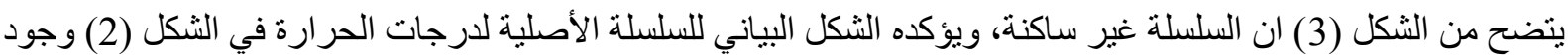



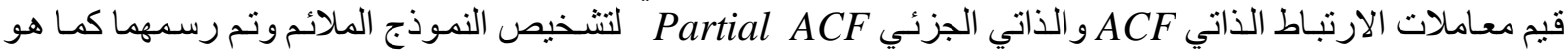

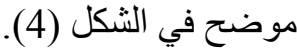

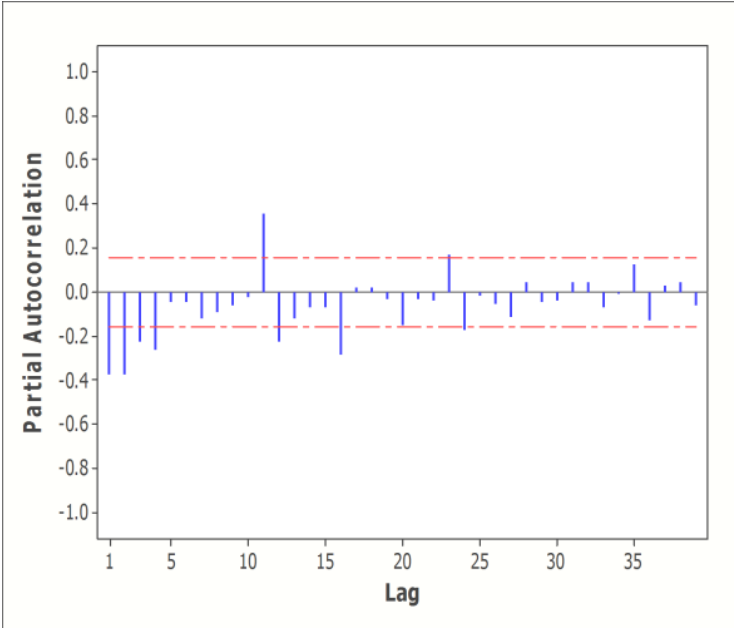

(ب)

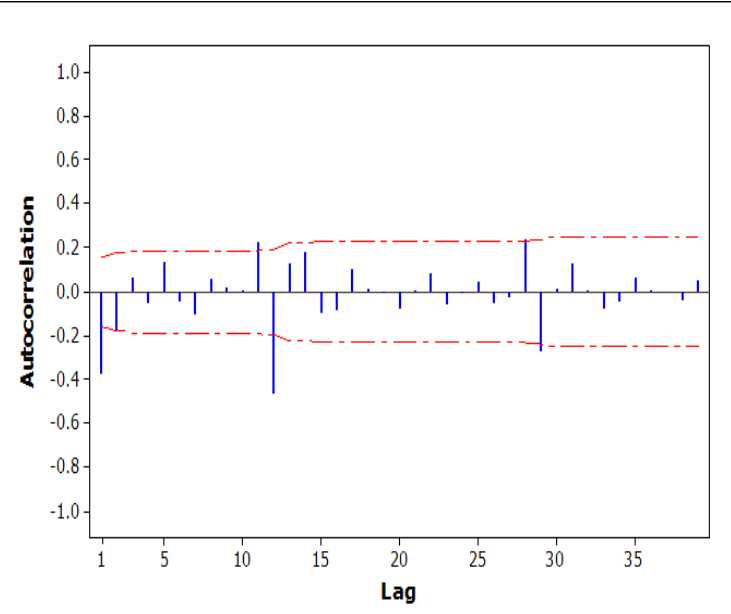

(l)

$$
\text { شكل (4) قيم معاملات الارتباط الذاتي ACF في (أ) و الذاتي الجزئي Partial ACF في (ب) بعد اخذ الفروق }
$$

بعد اخذ الفرق الأول و الفرق الموسمي للسلسلة الزمنية

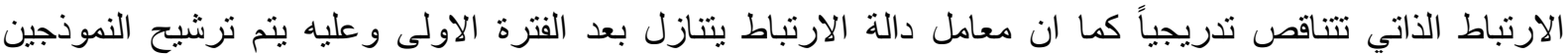

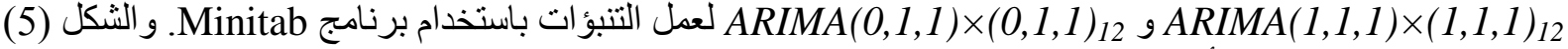
يوضح القيم الحقيقية والمتنبأ بها لدرجات الحر ارة للنموذجين المقترحين.

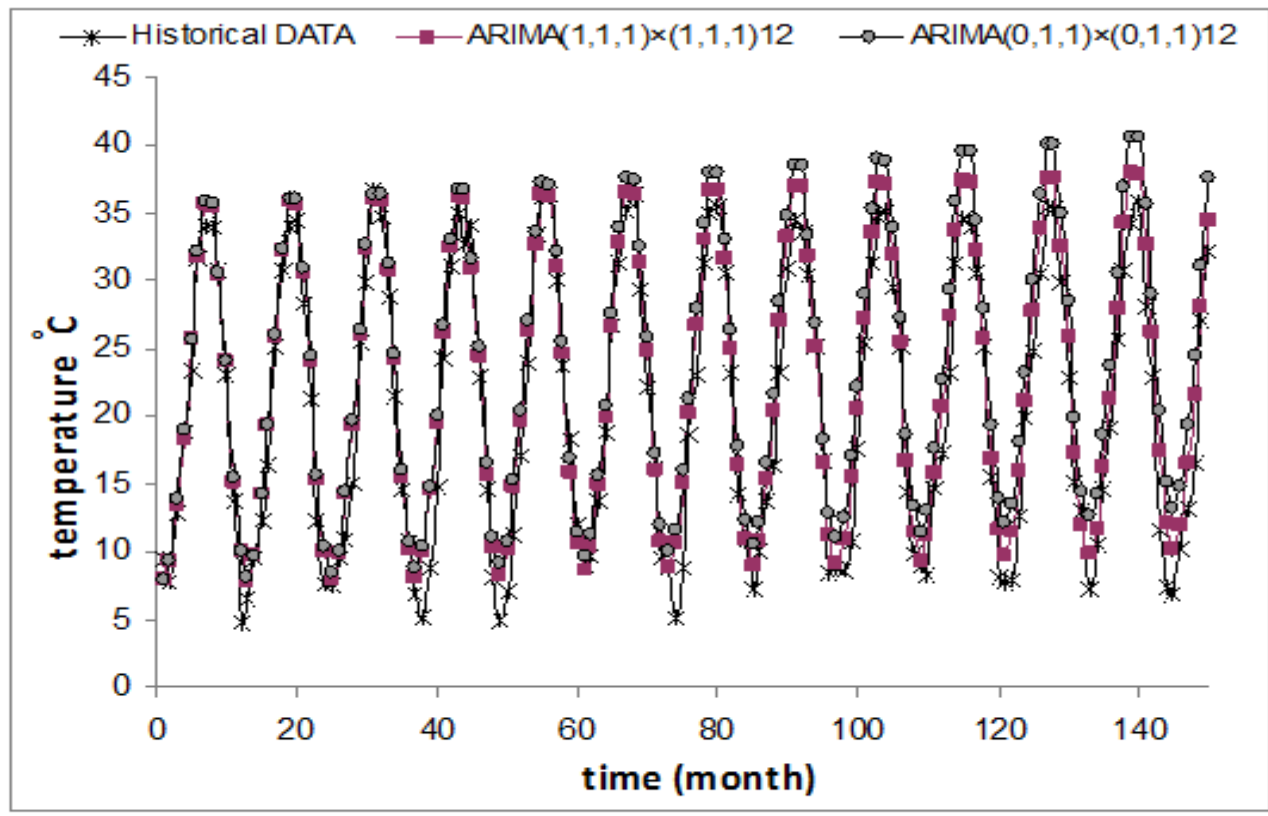

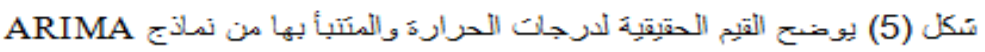




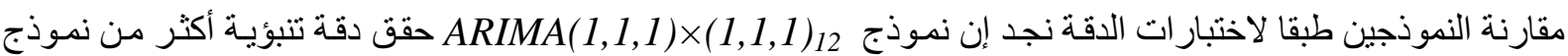
ARIMA $(0,1,1) \times\left(0,1,1\right.$ I $_{12}$

جدول (1) قيم اختبار ات الدقة التنبؤية لنماذج ARIMA المقترحة

\begin{tabular}{|c|c|c|}
\hline $\operatorname{ARIMA}(0,1,1) \times(0,1,1)_{12}$ & $\operatorname{ARIMA}(1,1,1) \times(1,1,1)_{12}$ & نوع الاختبار \\
\hline 69.134 & 15.280 & MSE \\
\hline 7.6449 & 3.5345 & MAE \\
\hline 0.5254 & 0.2555 & MAPE \\
\hline 4.8444 & 1.1932 & MPE \\
\hline
\end{tabular}



وقبـل استخدام النموذج لحسـاب التنبؤات

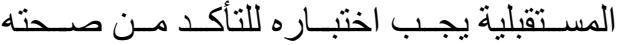

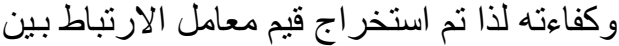



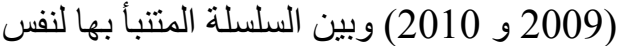

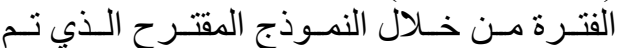

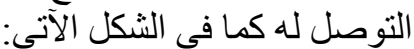

من الثـكل أعلاه يتضـح التقارب الكبير

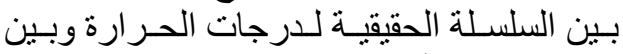

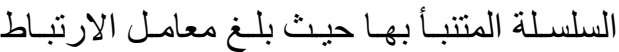



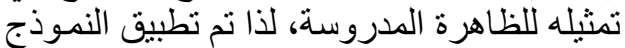

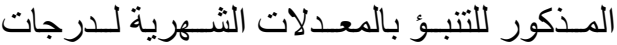

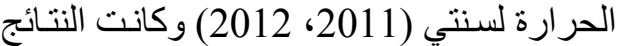

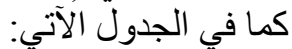

شكل (6) بيانات درجات الحرارة الثهرية الحقيقية و المتنبأ بها للسنتين (2009، 2010)

جدول (2) المعدلات الثهرية لدرجات الحرارة المتنبأ بها في منطقة الدر اسة وفق النموذج $\operatorname{ARIMA}(1,1,1) \times(1,1,1)_{12}$

\begin{tabular}{|c|c|c|c|}
\hline \multicolumn{2}{|c|}{ لسنة 2012} & \multicolumn{2}{|c|}{ لسنة 2011} \\
\hline درجة الحرارة & الثهر & درجة الحرارة & الشهر \\
\hline 9.21 & Jan & 9.43 & Jan \\
\hline 10.76 & Feb & 10.50 & Feb \\
\hline 14.81 & Mar & 14.24 & Mar \\
\hline 19.90 & Apr & 19.45 & Apr \\
\hline 26.66 & May & 26.47 & May \\
\hline 33.17 & Jun & 32.83 & Jun \\
\hline 36.61 & Jul & 36.34 & Jul \\
\hline 36.49 & Aug & 36.06 & Aug \\
\hline 31.58 & Sep & 30.9 & Sep \\
\hline 25.22 & Oct & 24.8 & Oct \\
\hline 16.84 & Nov & 15.77 & Nov \\
\hline 11.50 & Dec & 10.62 & Dec \\
\hline
\end{tabular}




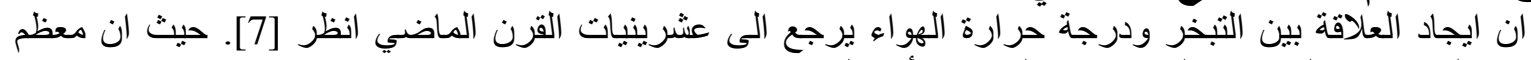

$$
\mathrm{ET}_{\mathrm{o}}=\mathrm{cT}^{\mathrm{a}}
$$

معادلات التبخر -نتح المعتمدة على درجات الحرارة تأخذ الصيغة الاتية [12]:

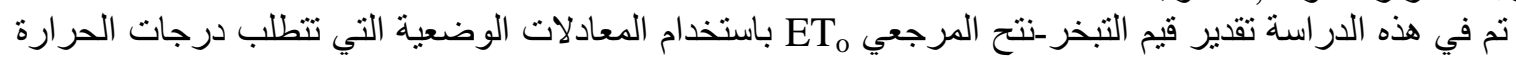
المتنبأ بها من نماذج ARIMA كمتغير ادخال بشكل اسأسي، وادناه الطرق الوضعية الني تم استخدامها في هذه الدراسة:

\section{1. طريقة بلاني كريديل Blaney-Criddle}

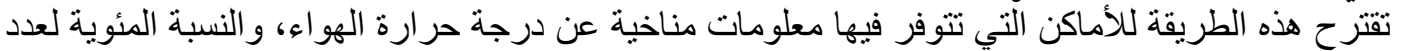

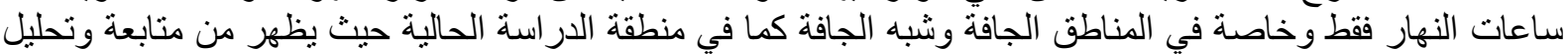

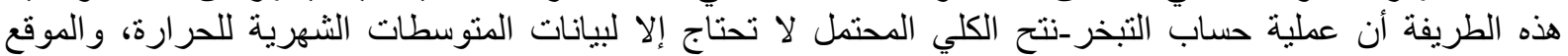

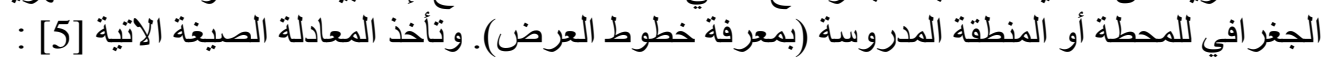
$\mathrm{ET}_{\mathrm{o}}=\mathrm{C}[\mathrm{P}(0.46 \mathrm{~T}+8.13)]$

(mm/day) :ETo

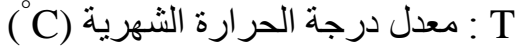

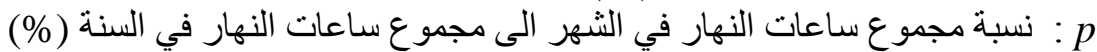
C : ثابت يعتمد على الرطوبة النسبية، عدد الساعات الني تكون فيها الثمس مشرقة، وسرعة الثرة الرياح خلال اليوم.[17]



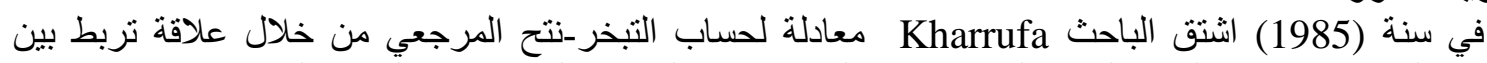

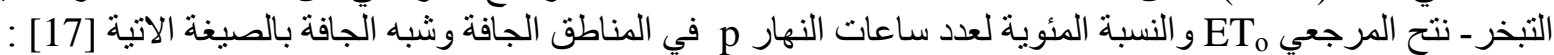
$\mathrm{ET}_{\mathrm{o}}=0.34 * \mathrm{p}^{*} \mathrm{~T}^{1.3}$



انتقق الباحث (1961) Hamon طريقة لحساب التبخر-نتح المرجعي بالاعتماد على درجة حرارة الهواء بالصيغة

$$
\begin{aligned}
& \mathrm{ET}_{\mathrm{o}}=0.55 * \mathrm{D}^{2 *} \mathrm{P}_{\mathrm{t}} \\
& \mathrm{P}_{\mathrm{t}}=4.95^{*} \mathrm{e}^{(0.062 \mathrm{~T})} / 100
\end{aligned}
$$

(16 : عدد ساعات سطوع الثمس. P : : تمثل كثافة بخار الماء المشبع وتحسب بالثكل الاتي:

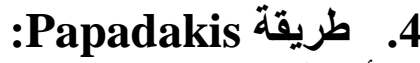

وتأخذ الصيغة الاتية [13] :

$\mathrm{ET}_{\mathrm{o}}=0.562 *\left(\mathrm{e}_{\mathrm{s}} \max -\left(\mathrm{e}_{\mathrm{s}} \min -2\right)\right)$

(pa) e ex min, e es max

يوضح الثكل(7) قيم التبخر-نتح المرجعي التي تم تقدير ها للسنتين (2011 و 2012) باستخدام طرق التبخر-نتح

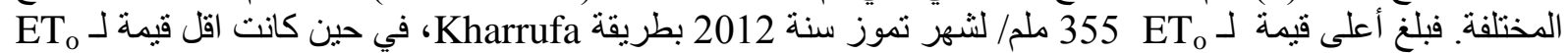

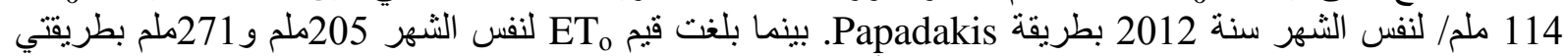

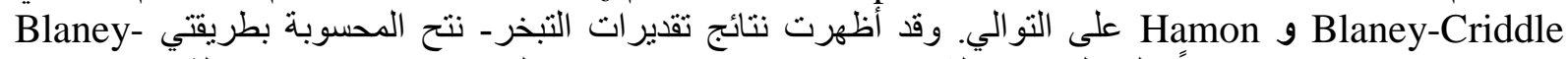

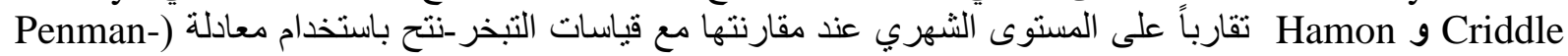

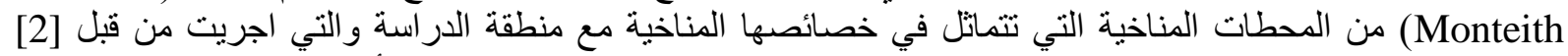






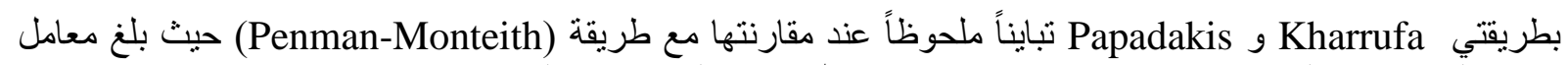
الارنباطً (0.89) بطريقة Papadakis. في حين كان معامل الارنباط (0.98) بطريقة Blaney-Criddle.

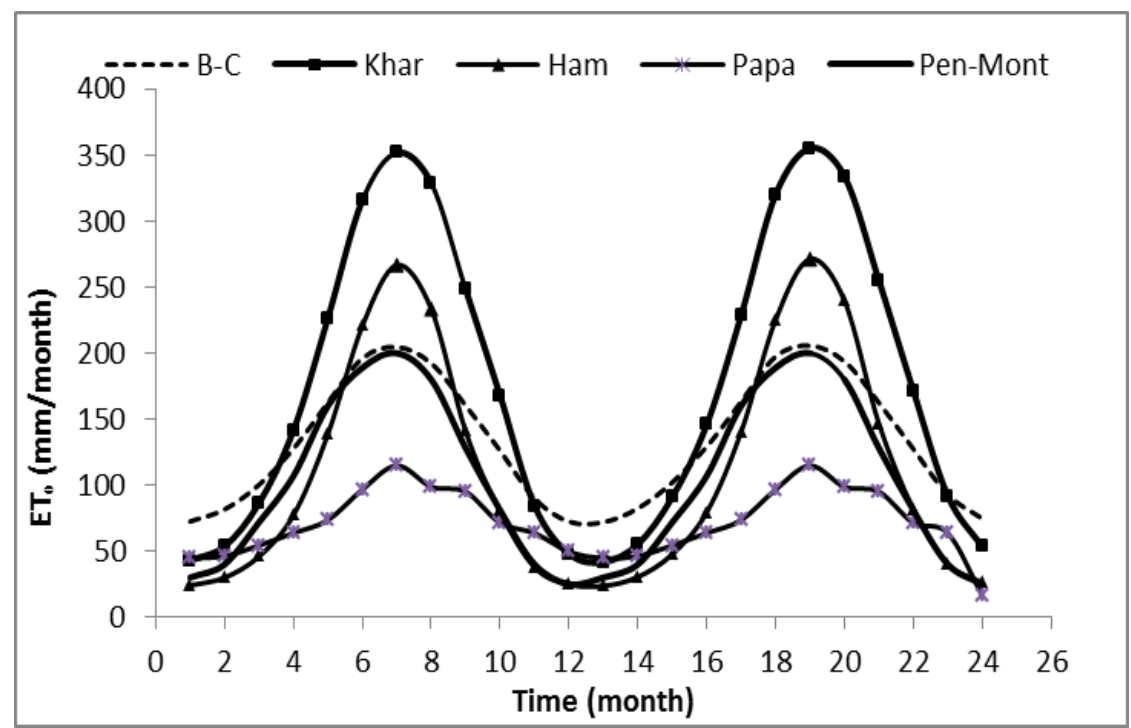

شكل (7) قيم التبخر-نتح المرجعي للسنتين (2011 و2012) بالطرق المختلفة مقارنة مع طريقة (بنمان-مونتث).

B-C=Blaney-Criddle; Khar=Kharrufa; Ham=Hamon; Papa=Papadakis; PenMont= Penman-Monteith

| الاستتناجاث:

1. من خلال تحليل السلسلة الزمنية باستخدام نموذج ARIMA الموسمي لغرض التنبؤ نستنتج إن درجات الحرارة الثهرية في منطقة الدر اسة تؤلف سلسلة زمنية غير ساكنة وموسمية اي تعيد نفسها كل 12 شهر أ وذللك من خلال قيم الترات معاملات الارتباط الذاتي.

2. عند مقارنة نماذج ARIMA المستخدمة في البحث تبين ان النموذج 12 (ORIMA(1,1,1)×(1,1,1) حقق قدرة تنبؤية

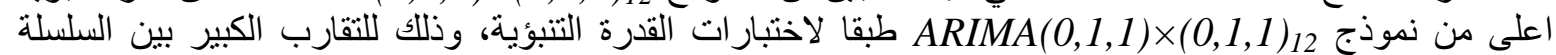

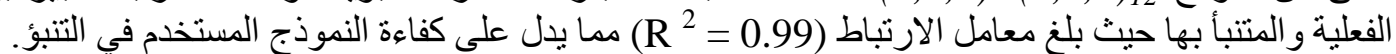

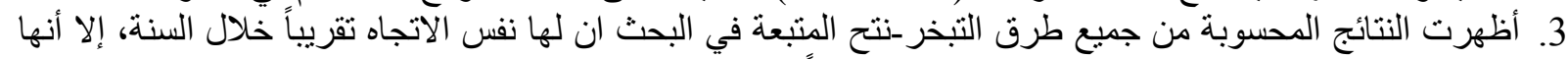

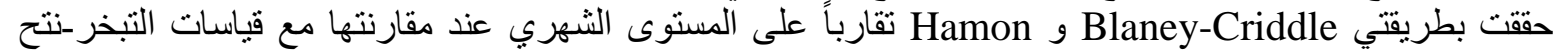

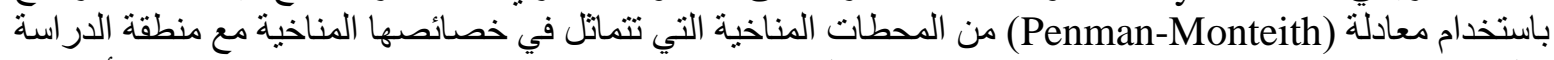

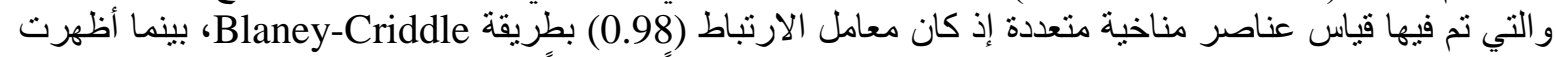
التقديرات المحسوبة بطريقتي Penman-) تبايناً ملحوظاً عند مقارنتها مع طريقة

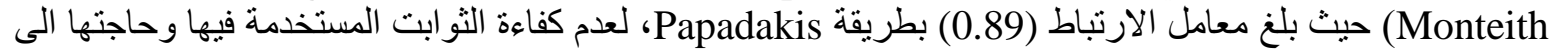
معايرة بما يتلاءم مع منطقة الدر اسة. 4. ان هنالك زيادة سنوية في مقدار التبخر-نتح المرجعي بنسبة (2011) في سنة سنة (2012) بالمقارنة مع سنة (2010)

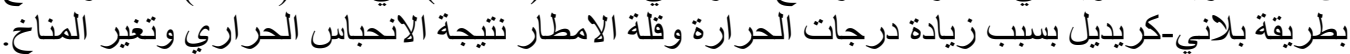

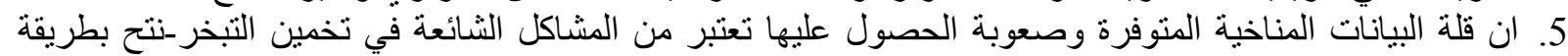
Penman-Monteith

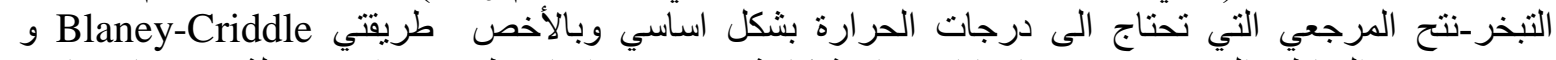
فamon سجلات التبخر-نتح التاريخية. 


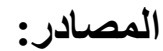

$$
\begin{aligned}
& \text { 1. إبر اهيم، بسام يونس. (2001). " التنبؤ بدرجات الحرارة في ولاية الخرطوم باستخدام أحد نماذج بوكس- جنكنس }
\end{aligned}
$$



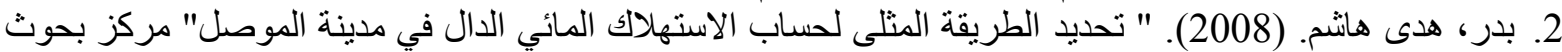

$$
\begin{aligned}
& \text { السدود و الموارد المائية، جامعة الموصل. }
\end{aligned}
$$

3. Blaney, H.F., Criddle, W.D. (1950)." Determining Water Requirements in Irrigated Areas from Climatological Irrigation Data". Technical Paper. No. 96, US Department of Agriculture, Soil Conservation Service, Washington, D.C.

4. Box, G., Jenkins, G., (1970). "Time Series Analysis, Forecasting and Control" San Francisco: Holden-Day.

5. Doorenbos, J., Pruitt, W.O., (1977). "Guidelines for predicting crop water requirements". FAO Irrigation and Drainage Paper 24, Food and Agriculture Organization of the United Nations, Rome.

6. Hamon, W.R., (1961). "Estimating potential evapotranspiration". Journal of Hydraulics Division, Proceedings of the American Society of Civil Engineers 871: 107-120.

7. Jensen, M.E., Burman, R.D., Allen, R.G., (1990). "Evapotranspiration and Irrigation Water Requirements". American Society of Civil Engineers: New York.

8. Keller, G., and Worrack, B., (1997). "Statistics for Management and Economics " Cole publishing Company, New York.

9. Kharrufa, N.S., (1985). "Simplified equation for evapotranspiration in arid regions". Beitragezur Hydrologic Sonderheft 5.1: 39-47.

10. Kosa, P. (2009). " Air Temperature and Actual Evapotranspiration Correlation Using Landsat 5 TM Satellite Imagery" Kasetsart Journal. (Nat.Sci.) 43 : 605 - 611

11. Linacre, E.T. (1977). "A simple formula for estimating evaporation rates in various climates, using temperature data alone". Agricultural Meteorology, 18: 409-424

12. Mishra, A. K., Desai, V. R., and Singh, V.P.,(2007). "Drought Forecasting Using a Hybrid Stochastic and Neural Network Model". Journal of Hydrologic Engineering, Vol. 12, No. 6, ASCE.

13. Rosenberry, O. D., Thomas, C. W., Donald, C. B., Gene, E. L., (2007) " Comparison of 15 evaporation methods applied to a small mountain lake in the northeastern USA". Journal of Hydrology, 340, 149- 166.

14. See, L., and Openshaw, S., (1998). "Using Soft Computing Techniques to Enhance Flood Forecasting on The River Ouse". Proceeding Hydroinformatics'98: 3rd International Conference on Hydro informatics, Copenhagen, Denmark, 24-26 August, pp. 819-824.

15. Vandaele, W. (1983). "Applied Time Series and Box-Jenkins Models", John Wiley \& Sons.

16. Venema, H.D., Schiller, E.J., and Adamowski, K. (1996) "Evidence of Climate Change in The Senegal River Basin", Water Resources Development, 12, 531-546

17. Xu, C. Y., and Singh, V. P., (2001). " Evaluation and generalization of temperature-based methods for calculating evaporation" Hydro. Process, 15, 305-319.

18. Yurekli, K. and Kurunc, A. (2006). " Performances of Stochastic Approaches in Generating Low Streamflow Data for Drought Analysis" Journal of Spatial Hydrology Spring, Vol. 5, No. 1.

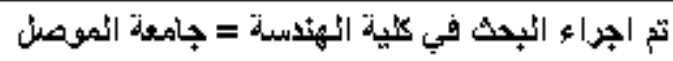

